\title{
Gauge Mediation of Supersymmetry Breaking and the Froggatt-Nielsen Mechanism
}

\author{
Zhong-hua Zhang and Da-Xin Zhang \\ School of Physics and State Key Laboratory of Nuclear Physics and Technology, \\ Peking University, Beijing 100871, China
}

\begin{abstract}
In the model of gauge mediation of SUSY breaking in the presence of tree-level mediation, the Froggatt-Nielsen mechanism provides a different hierarchy of sparticle masses. We study the spectra and show the results to be like those in an effective supersymmetric model.
\end{abstract}

Supersymmetry (SUSY) is an important candidate for the physics beyond the Standard Model (SM). Among many models of SUSY breaking, gauge mediation of SUSY breaking (GMSB) [1, 2, 3, 4, 5] provides an important mechanism. In the usual GMSB models, SUSY is broken in a hidden sector and the effects of SUSY breaking are transmitted to the visible sector by gauge interactions of the SM gauge groups. It was noticed in [6] that the effects of SUSY breaking can be extended in the presence of extra gauge interactions. In [6] this extra gauge group is a $\mathrm{U}(1)$-subgroup of $\mathrm{SO}(10)$ in the grand unification theory (GUT) and it is broken at around the unification scale. Consequently, SUSY particles receive tree-level SUSY breaking effects mediated by the extra $\mathrm{U}(1)$ gauge interactions if these particles carry the $\mathrm{U}(1)$ quantum numbers. The supertrace formula constrains the total sfermion and the corresponding fermion total squared masses to be the same [7]. In this paper, the supertrace formula can be satisfied by compensating the contribution from MSSM particles by the heavy particles of the GUT scale [6].

Here we will study the possibility that the extra gauged $U(1)$ is the flavor symmetry of the Froggatt-Nielsen mechanism (FNM) [, 9] such that the mechanisms of SUSY breaking and of generation of fermion masses have a common origin. Different from the model of [6], the quantum numbers and consequently the spectra of the SUSY particles are very different. There 
are superfields which do not carry the $\mathrm{U}(1)_{F N}$ quantum numbers, their scalar components get masses at two-loop level.

In the literature, combining FNM and GMSB has been studied in [10] where SUSY is broken at low energy $(\sim 100 \mathrm{TeV})$. Unlike the original FNM where extra (super-)fields are at the GUT or even higher scale, in [10] the extra superfields are introduced at the low energy scale and they mix with those in the minimal supersymmetric standard model (MSSM), especially in the fermion sector. Consequently, unitarity of the $3 \times 3$ quark mixing matrix is violated, which makes the model rather complicated [11. Different from [10], in the present work the extra superfields are introduced at around the GUT scale as in the original FNM. At low energy the model is reduced to be the MSSM, thus the possible problem of unitarity violation is absent.

We will firstly present the model. Then we will classify the mechanism for generating the SUSY breaking parameters. Finally we will present the spectra and summarize.

\section{2}

The model extends the SM gauge group by adding a gauged $\mathrm{U}(1)_{F N}$. Instead of being a generator of GUT group, we take this $\mathrm{U}(1)_{F N}$ to be irrelevant with unification as there exists no evidence of any relevance. The GUT group is taken to be SU(5), although a larger group is also possible.

At around the GUT scale we add in extra Higgs-like superfields and matter-like superfields. The Higgs-like superfields are those complete representations of SU(5) as in the original model in [8, 9], extended by a $\mathrm{SU}(5)$ singlet sector. Besides the possibly existed colored Higgs whose effects are calculable, the newly introduced Higgs-like superfields will not affect gauge coupling unification. The singlet sector contains at least two effective superfields: $N^{\prime}$ whose F-component has a nonzero vacuum expectation value (VEV) which breaks SUSY,

$$
\left\langle N^{\prime}\right\rangle=F \theta^{2}
$$

and $N$ whose VEV breaks the $\mathrm{U}(1)_{F N}$,

$$
\langle N\rangle=M \sim M_{G U T}
$$

The $\mathrm{U}(1)_{F N}$ charges of both $N^{\prime}$ and $N$ are taken to be 5 for convenience. Note that we do not care if they are elementary or composite.

The extra heavy matter-like superfields form complete representations of the GUT SU(5) group so as not to spoil the gauge coupling unification. For those which are relevant to SUSY breaking, They are taken to be vector-like $F+\bar{F}=5+\overline{5}, 10+\overline{10}+\ldots$. We take their $\mathrm{U}(1)_{F N}$ charges to be -3 for $F$ and -2 for $\bar{F}$. The superpotential of this heavy sector is

$$
W=h N F \bar{F}+h^{\prime} N^{\prime} F \bar{F}
$$


The remaining chiral superfields are those in the MSSM. Their U(1) FN charges 1 are,

$$
\begin{array}{r}
A_{Q_{1}, Q_{2}, Q_{3}}=4,2,0, \quad A_{\bar{u}_{1}, \bar{u}_{2}, \bar{u}_{3}}=4,2,0, \quad A_{\bar{d}_{1}, \bar{d}_{2}, \bar{d}_{3}}=1+s, s, s, \\
A_{L_{1}, L_{2}, L_{3}}=1+s, s, s, \quad A_{\bar{e}_{1}, \bar{e}_{2}, \bar{e}_{3}}=4,2,0, \quad A_{H_{u}}=A_{H_{d}}=0,
\end{array}
$$

as in the original FNM. The integer $s$ is allowed to take 0,1 or 2 , corresponding to $\tan \beta$ taking a large, middle or small value. Note that there exist no direct couplings between MSSM superfields and $N$ or $N^{\prime}$ so that at low energy MSSM is recovered.

\section{3}

In the presence of the nonzero VEV in (1), the vector superfield of the $\mathrm{U}(1)_{F N}$ gauge group develops an effective D-component as

$$
<D_{F N}>=2 g A_{N^{\prime}}\left(\frac{F}{M_{V}}\right)^{2}
$$

where $M_{V}$ is the mass of the gauge field of the $\mathrm{U}(1)_{F N}$ group. The other heavy superfields are the $F+\bar{F}$ 's, they receive SUSY breaking effect through (3).

The effects of SUSY breaking are transmitted to the MSSM sector in the following ways:

(1) The scalars of the MSSM chiral superfields which carry the $\mathrm{U}(1)_{F N}$ charges get masses at tree-level through couplings to the $\mathrm{U}(1)_{F N}$ vector superfield,

$$
\tilde{m}_{F}^{2}=\frac{A_{F}}{2 A_{N}} m^{2}, \quad m \equiv \frac{F}{M} .
$$

(2) The gauginos of the MSSM acquire masses in the same way as in the usual GMSB model, where again the heavy $F+\bar{F}$ play the roles of messengers,

$$
\begin{array}{r}
M_{a}=\frac{\alpha_{a}}{4 \pi} m \sum_{i, j} \frac{h_{i j}^{\prime}}{h_{i j}} n_{a}(i, j) g\left(x_{i j}\right), \quad x_{i j}=\frac{h_{i j}^{\prime} F}{h_{i j} M}, \quad a=1,2,3, \\
g(x)=\frac{1}{x^{2}}[(1+x) \log (1+x)+(1-x) \log (1-x)] .
\end{array}
$$

(3) The scalars of the MSSM chiral superfields which do not carry the $\mathrm{U}(1)_{F N}$ charges get masses at 2-loop level using the same mechanism as in the usual model of GMSB, where the heavy $F+\bar{F}$ play the roles of messengers,

$$
\tilde{m}^{2}(Q)_{2 l o o p}=2 \eta \sum_{a} c_{a}(Q) M_{a}^{2}, \quad \eta=\frac{\sum\left(h_{i}^{\prime} / h_{i}\right)^{2}}{\left(\sum_{i} h_{i}^{\prime} / h_{i}\right)^{2}} \geq \frac{1}{3} .
$$

At the same time, those scalars in (7) receive corrections (10), accordingly.

\footnotetext{
${ }^{1}$ In some FN models the charges of $\mathrm{u}$ and e contain parameters $\mathrm{p}$ and $\mathrm{s}$ respectively, which can be different or the same. However, in our scheme, the $U(1)_{F N}$ anomalies must be canceled by the Green-Schwarz mechanism 12 13, 14, which requires $\mathrm{p}=\mathrm{s}$ and that the $\mathrm{FN}$ charges in the same $\mathrm{SU}(5)$ multiplet should be the same.
} 
Now we are ready to calculate the spectrum in the MSSM. We will first input the value $F$, $M, \Lambda_{G U T}$. Then we will take eq.(10) as initial values at the GUT scale. Finally, we will use the renormalization group equations to run the scale down to the electroweak scale. The final step will be done with the help of Suspect 2.41[15].

Numerically the unified gaugino mass is taken as $M_{1 / 2}=300 \mathrm{Gev}$. For the typical scale of sparticle mass we take $m=3.2 \mathrm{TeV}$. The lightest neural Higgs mass is taken to satisfy the bound $m_{h}>114 \mathrm{GeV}$. We take $s=0,1,2$ for $\tan \beta=30,20,9$, respectively. We give these spectra in Table 1, 2, 3, respectively.

We find that the NLSP is always a Bino-like neutralino whose mass is around $125 \mathrm{GeV}$. The mass of the lighter chargino is around $230 \mathrm{GeV}$, which is very different from the result of nearly degeneration of lightest neutralino and the lighter chargino in [6]. These results imply that they can be easily produced and identified in the LHC experiments. We find, also different from the results of [6] where all the sfermions are heavy, that the sfermions for the first two generations are usually heavy, corresponding to the effective SUSY scenario 16 of suppressing FCNC. The sfermions of the third generation are generally lighter than $1 \mathrm{TeV}$, making them accessible at the LHC experiments. To be intuitive, we also plot an example in Fig. 1.

\section{5}

In summery, we have studied the spectra in the model of GMSB with FNM. We find the results are very different from those in [6]. No tree-level FCNC is introduced due to a high energy scale of SUSY breaking, which is different from the case in [10, 11.

This work was supported in part by the National Natural Science Foundation of China (NSFC) under the grant No. 10435040.

\section{References}

[1] M. Dine, W. Fischler, and M. Srednicki, Nucl. Phys. B189, 575(1981);

[2] M. Dine and W. Fischler, Phys. Lett. 110B, 227(1982);

[3] M. Dine and A. E. Nelson, Phys. Rev. D48, 1277(1993) arXiv:hep-ph/9303230;

[4] M. Dine, A. E. Nelson and Y. Shirman, Phys. Rev. D51, 1362(1995) [arXiv:hep-ph/9408384;

[5] M. Dine, A. E. Nelson, Y. Nir and Y. Shirman, Phys. Rev. D53, 2658(1996) arXiv:hep-ph/9507378.

[6] M. Nardecchia, A. Romanino and R. Ziegler, JHEP 0911, 112(2009). [arXiv:hep$\mathrm{ph} / 0909.3058 \mathrm{v} 2]$.

[7] S. Ferrara, L. Girardello and F. Palumbo Phys. Rev. D20, 403(1979)

[8] C. D. Froggatt and H. B. Nielsen, Nucl. Phys. B147, 277(1979). 


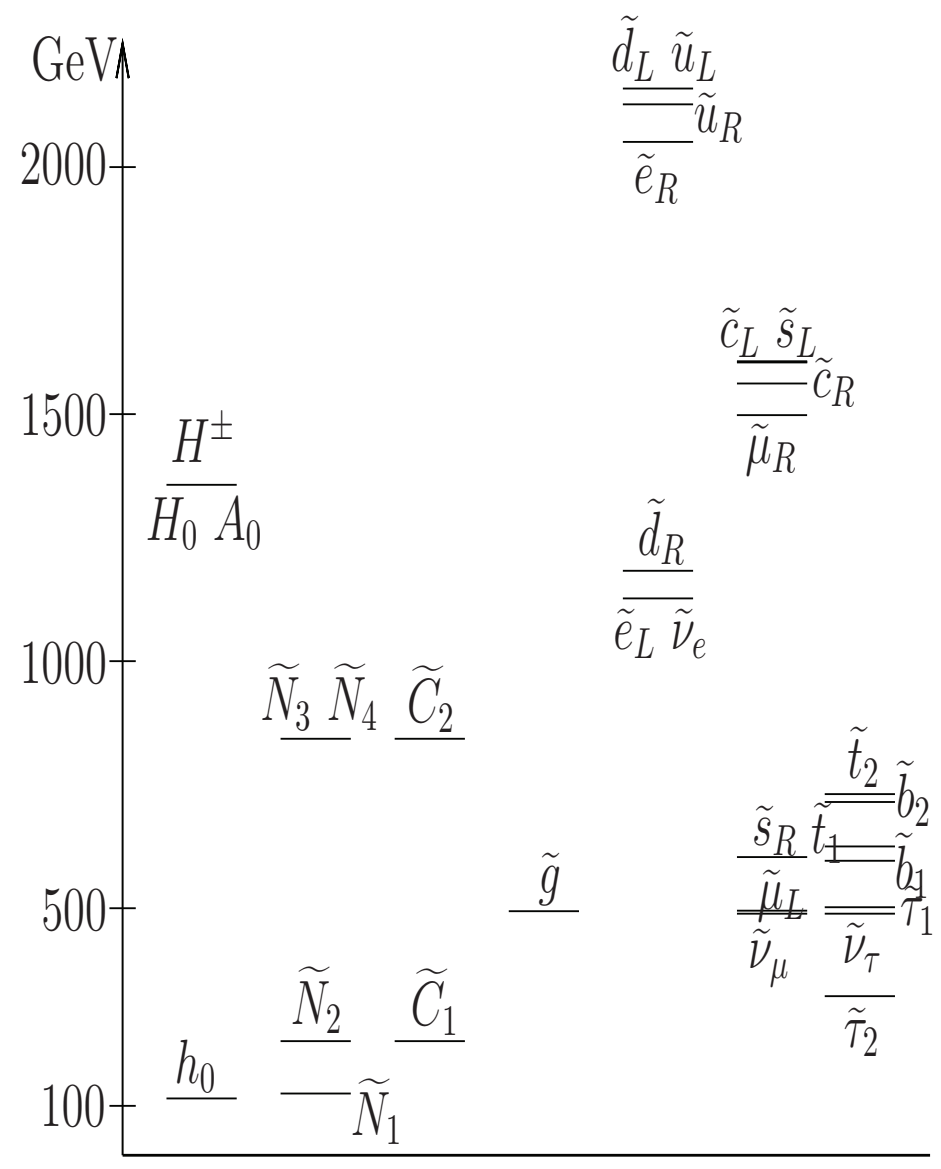

Figure 1: An example of spectrum, corresponding to $m=3.2 \mathrm{TeV}, M_{1 / 2}=300 \mathrm{Gev}, \tan \beta=$ $30, s=0, \operatorname{sign}(\mu)=+, A=0, \eta=1$. All the masses are in $\mathrm{GeV}$.

[9] For a recent review, see K. S. Babu, "TASI Lectures on Flavor Physics", [arXiv:hep$\mathrm{ph} / 0910.2948 \mathrm{v} 1]$

[10] D. E. Kaplan, F. Lepeintre, A. Masiero, A. E. Nelson and A. Riotto, Phys. Rev. D60, 055003(1999) arXiv:hep-ph/9806430.

[11] D. E. Kaplan and G. D. Kribs, Phys. Rev. D61, 075011(2000) arXiv:hep-ph/9906341.

[12] M.B. Green and J.H. Schwarz, Phys. Lett. B149, 117(1984);

[13] M.B.Green, J.H. Schwarz and P. West, Nucl. Phys. B254, 327(1985).

[14] M.B. Green and J.H. Schwarz, Nucl. Phys. B255, 93(1985);

[15] A. Djouadi, J. L. Kneur and G. Moultaka, "SuSpect: A Fortran code for the supersymmetric and Higgs particle spectrum in the MSSM," Comput. Phys. Commun. 176, 426(2007) 
arXiv:hep-ph/0211331,

http://www.lpta.univ-montp2.fr/users/kneur/Suspect/.

[16] A.G. Cohen, D.B. Kaplan and A.E. Nelson, Phys. Lett. B388, 588(1996). arXiv:hep-ph/9607394 v2]. 


\begin{tabular}{|c|c|c|c|c|}
\hline Names & & $\mathrm{s}=0$ & $\mathrm{~s}=1$ & $\mathrm{~s}=2$ \\
\hline \multirow[t]{4}{*}{ Higgs: } & $m_{h^{0}}$ & 115.0 & 115.1 & 115.2 \\
\hline & $m_{H^{0}}$ & 1357 & 1357 & 1357 \\
\hline & $m_{A}$ & 1357 & 1357 & 1357 \\
\hline & $m_{H^{ \pm}}$ & 1359 & 1359 & 1359 \\
\hline Gluinos: & $M_{\tilde{g}}$ & 494.0 & 501.6 & 505.4 \\
\hline \multirow[t]{4}{*}{ Neutralinos: } & $m_{\chi_{1}^{0}}$ & 125.4 & 125.4 & 125.4 \\
\hline & $m_{\chi_{2}^{0}}$ & 231 & 231 & 231 \\
\hline & $m_{\chi_{3}^{0}}$ & 840 & 840 & 840 \\
\hline & $m_{\chi_{4}^{0}}$ & 843 & 843 & 843 \\
\hline \multirow[t]{2}{*}{ Charginos: } & $m_{\chi_{1}^{ \pm}}$ & 231 & 231 & 231 \\
\hline & $m_{\chi_{2}^{ \pm}}$ & 844 & 844 & 844 \\
\hline \multirow[t]{12}{*}{ Squarks: } & $m_{\tilde{u}_{L}}$ & 2159 & 2159 & 2159 \\
\hline & $m_{\tilde{u}_{R}}$ & 2127 & 2127 & 2127 \\
\hline & $m_{\tilde{d}_{L}}$ & 2160 & 2160 & 2160 \\
\hline & $m_{\tilde{d}_{R}}$ & 1183 & 1183 & 1183 \\
\hline & $m_{\tilde{c}_{L}}$ & 1605 & 1605 & 1605 \\
\hline & $m_{\tilde{c}_{R}}$ & 1562 & 1562 & 1562 \\
\hline & $m_{\tilde{s}_{L}}$ & 1607 & 1607 & 1607 \\
\hline & $m_{\tilde{s}_{R}}$ & 604 & 1183 & 1562 \\
\hline & $m_{\tilde{t}_{1}}$ & 625 & 627 & 630 \\
\hline & $m_{\tilde{t}_{2}}$ & 731 & 732 & 733 \\
\hline & $m_{\tilde{b}_{1}}$ & 596 & 706 & 707 \\
\hline & $m_{\tilde{b}_{2}}$ & 715 & 1185 & 1563 \\
\hline \multirow[t]{9}{*}{ Sleptons: } & $m_{\tilde{e}_{L}}$ & 1127 & 1514 & 1821 \\
\hline & $m_{\tilde{e}_{R}}$ & 2051 & 2051 & 2051 \\
\hline & $m_{\tilde{\mu}_{1}}$ & 495 & 1127 & 1514 \\
\hline & $m_{\tilde{\mu}_{2}}$ & 1469 & 1469 & 1469 \\
\hline & $m_{\tilde{\tau}_{1}}$ & 322 & 329 & 330 \\
\hline & $m_{\tilde{\tau}_{2}}$ & 502 & 1127 & 1515 \\
\hline & $m_{\tilde{\nu}_{e}}$ & 1124 & 1512 & 1820 \\
\hline & $m_{\tilde{\nu}_{\mu}}$ & 489 & 1124 & 1512 \\
\hline & $m_{\tilde{\nu}_{\tau}}$ & 489 & 1124 & 1512 \\
\hline
\end{tabular}

Table 1: The spectra corresponding to $\tan \beta=30$, with $\mathrm{s}=0,1,2$ respectively. All the masses are in $\mathrm{GeV}$. 


\begin{tabular}{|c|c|c|c|c|}
\hline Names & & $\mathrm{s}=0$ & $\mathrm{~s}=1$ & $\mathrm{~s}=2$ \\
\hline \multirow[t]{4}{*}{ Higgs: } & $\bar{m}_{h^{0}}$ & 114.5 & 114.6 & 114.6 \\
\hline & $m_{H^{0}}$ & 1356.5 & 1356.5 & 1356.5 \\
\hline & $m_{A}$ & 1356.5 & 1356.5 & 1356.5 \\
\hline & $m_{H^{ \pm}}$ & 1359 & 1359 & 1359 \\
\hline Gluinos: & $M_{\tilde{g}}$ & 494.0 & 502.0 & 505.4 \\
\hline \multirow[t]{4}{*}{ Neutralinos: } & $m_{\chi_{1}^{0}}$ & 125 & 125 & 125 \\
\hline & $m_{\chi_{2}^{0}}$ & 230.4 & 230.4 & 230.4 \\
\hline & $m_{\chi_{3}^{0}}$ & 839.5 & 839.5 & 839.5 \\
\hline & $m_{\chi_{4}^{0}}$ & 843 & 843 & 843 \\
\hline \multirow[t]{2}{*}{ Charginos: } & $m_{\chi_{1}^{ \pm}}$ & 230 & 230 & 230 \\
\hline & $m_{\chi_{2}^{ \pm}}$ & 844 & 844 & 844 \\
\hline \multirow[t]{12}{*}{ Squarks: } & $m_{\tilde{u}_{L}}$ & 2159 & 2159 & 2159 \\
\hline & $m_{\tilde{u}_{R}}$ & 2127 & 2127 & 2127 \\
\hline & $m_{\tilde{d}_{L}}$ & 2160 & 2160 & 2160 \\
\hline & $m_{\tilde{d}_{R}}$ & 1183 & 1562 & 1866 \\
\hline & $m_{\tilde{c}_{L}}$ & 1605 & 1605 & 1605 \\
\hline & $m_{\tilde{c}_{R}}$ & 1562 & 1562 & 1562 \\
\hline & $m_{\tilde{s}_{L}}$ & 1607 & 1607 & 1607 \\
\hline & $m_{\tilde{s}_{R}}$ & 604 & 1183 & 1562 \\
\hline & $m_{\tilde{t}_{1}}$ & 625 & 627 & 630 \\
\hline & $m_{\tilde{t}_{2}}$ & 732 & 732 & 733 \\
\hline & $m_{\tilde{b}_{1}}$ & 599 & 708 & 708 \\
\hline & $m_{\tilde{b}_{2}}$ & 713 & 1183 & 1562 \\
\hline \multirow[t]{9}{*}{ Sleptons: } & $m_{\tilde{e}_{L}}$ & 1127 & 1514 & 1821 \\
\hline & $m_{\tilde{e}_{R}}$ & 2051 & 2051 & 2051 \\
\hline & $m_{\tilde{\mu}_{1}}$ & 495 & 1127 & 1514 \\
\hline & $m_{\tilde{\mu}_{2}}$ & 1469 & 1469 & 1469 \\
\hline & $m_{\tilde{\tau}_{1}}$ & 326 & 331 & 331 \\
\hline & $m_{\tilde{\tau}_{2}}$ & 499 & 1127 & 1514 \\
\hline & $m_{\tilde{\nu}_{e}}$ & 1124 & 1512 & 1820 \\
\hline & $m_{\tilde{\nu}_{\mu}}$ & 489 & 1124 & 1512 \\
\hline & $m_{\tilde{\nu}_{\tau}}$ & 489 & 1124 & 1512 \\
\hline
\end{tabular}

Table 2: The spectra corresponding to $\tan \beta=15$, with $\mathrm{s}=0,1,2$ respectively. All the masses are in $\mathrm{GeV}$. 


\begin{tabular}{|c|c|c|c|c|}
\hline Names & & $\mathrm{s}=0$ & $\mathrm{~s}=1$ & $\mathrm{~s}=2$ \\
\hline \multirow[t]{4}{*}{ "Higgs: } & $m_{h_{h^{0}}}$ & "113.4 & 113.5 & 113.5 \\
\hline & $m_{H^{0}}$ & 1356.7 & 1356.7 & 1356.7 \\
\hline & $m_{A}$ & 1356.7 & 1356.7 & 1356.7 \\
\hline & $m_{H^{ \pm}}$ & 1359 & 1359 & 1359 \\
\hline Gluinos: & $M_{\tilde{g}}$ & 493.9 & 501.5 & 505.4 \\
\hline \multirow[t]{4}{*}{ Neutralinos: } & $m_{\chi_{1}^{0}}$ & 125 & 125 & 125 \\
\hline & $m_{\chi_{2}^{0}}$ & 229.7 & 229.7 & 229.7 \\
\hline & $m_{\chi_{3}^{0}}$ & 839 & 839 & 839 \\
\hline & $m_{\chi_{4}^{0}}$ & 844 & 844 & 844 \\
\hline \multirow[t]{2}{*}{ Charginos: } & $m_{\chi_{1}^{ \pm}}$ & 229.7 & 229.7 & 229.7 \\
\hline & $m_{\chi_{2}^{ \pm}}$ & 844.6 & 844.6 & 844.6 \\
\hline \multirow[t]{12}{*}{ Squarks: } & $m_{\tilde{u}_{L}}$ & 2159 & 2159 & 2159 \\
\hline & $m_{\tilde{u}_{R}}$ & 2127 & 2127 & 2127 \\
\hline & $m_{\tilde{d}_{L}}$ & 2160 & 2160 & 2160 \\
\hline & $m_{\tilde{d}_{R}}$ & 1183 & 1562 & 1866 \\
\hline & $m_{\tilde{c}_{L}}$ & 1605 & 1605 & 1605 \\
\hline & $m_{\tilde{c}_{R}}$ & 1562 & 1562 & 1562 \\
\hline & $m_{\tilde{s}_{L}}$ & 1607 & 1607 & 1607 \\
\hline & $m_{\tilde{s}_{R}}$ & 604 & 1183 & 1562 \\
\hline & $m_{\tilde{t}_{1}}$ & 623 & 625 & 628 \\
\hline & $m_{\tilde{t}_{2}}$ & 733 & 733 & 733 \\
\hline & $m_{\tilde{b}_{1}}$ & 602 & 709 & 709 \\
\hline & $m_{\tilde{b}_{2}}$ & 710 & 1183 & 1562 \\
\hline \multirow[t]{9}{*}{ Sleptons: } & $m_{\tilde{e}_{L}}$ & 1127 & 1514 & 1821 \\
\hline & $m_{\tilde{e}_{R}}$ & 2051 & 2051 & 2051 \\
\hline & $m_{\tilde{\mu}_{1}}$ & 489 & 1127 & 1514 \\
\hline & $m_{\tilde{\mu}_{2}}$ & 1469 & 1469 & 1469 \\
\hline & $m_{\tilde{\tau}_{1}}$ & 330 & 331 & 331 \\
\hline & $m_{\tilde{\tau}_{2}}$ & 496 & 1127 & 1514 \\
\hline & $m_{\tilde{\nu}_{e}}$ & 1124 & 1512 & 1820 \\
\hline & $m_{\tilde{\nu}_{\mu}}$ & 489 & 1124 & 1512 \\
\hline & $m_{\tilde{\nu}_{\tau}}$ & 489 & 1124 & 1512 \\
\hline
\end{tabular}

Table 3: The spectra corresponding to $\tan \beta=9$, with $\mathrm{s}=0,1,2$ respectively. All the masses are in $\mathrm{GeV}$. 